\title{
Short communication \\ Are current drug development programmes realising the full potential of new agents? The scenario
}

\author{
Stephen RD Johnston
}

Department of Medicine, Royal Marsden NHS Foundation Trust, Chelsea, London SW3 6JJ, UK

Corresponding author: Stephen RD Johnston, stephen.johnston@rmh.nhs.uk

Published: 18 December 2009

This article is online at

http://breast-cancer-research.com/supplements/11/S3/S21
Breast Cancer Research 2009, 11(Suppl 3):S21

(doi:10.1186/bcr2440)

(c) 2009 BioMed Central Ltd

\section{Introduction}

Drug development for human cancer is a lengthy and costly business, and many potentially promising therapeutics never make it to the clinic - yet in breast cancer, the past 10 to 15 years have seen the introduction of several new cytotoxic agents (paclitaxel, docetaxel, capecitabine and vinorelbine), two new classes of endocrine therapies (the aromatase inhibitors letrozole, anastrozole and exemestane; and the pure oestrogen receptor (ER) antagonist fulvestrant), and most recently targeted biological therapies against the human epidermal growth factor receptor-2 (HER2) growth factor receptor (trastuzumab, lapatinib) and vascular endothelial growth factor (bevacizumab). For each drug, the clinical development process has spanned a minimum of 10 to 15 years, culminating in large expensive pivotal registration trials, each costing millions of dollars in research and development expenditure.

While the incremental benefit provided by each new therapy in advanced breast cancer may be relatively small, three of these therapies (taxanes, aromatase inhibitors, trastuzumab) have now been incorporated into adjuvant therapies in early breast cancer where they are likely to make a much greater impact on survival in this disease. While these are indeed notable success stories, the challenges now faced by the next generation of molecularly targeted therapeutics are substantial.

\section{Principles for successful drug development with targeted therapies}

To date the most significant developments in the systemic treatment of breast cancer have been from therapies targeted against the ER and HER2. Their development pathway has been lengthy, however, with several decades passing from the first discovery of the ER, and then HER2, to the results of large-scale adjuvant trials in appropriate patients that demonstrated improved overall clinical outcomes [1-3]. Despite this timescale, however, the key principles that have underpinned their eventual successful development need to be understood, as these remain highly relevant to modern targeted therapies.

Firstly, it is crucial to show that any gene or protein product for any potential new target is implicated in the pathogenesis of the disease. Not only may this be as a significant prognostic factor, but also as predictive factor such that inhibiting or neutralising the function of the given gene/ protein product will yield a significant anti-cancer effect. Secondly, a robust and reliable assay needs to be established to measure expression of the target in human breast carcinomas, and this assay needs to be easily reproducible to good laboratory practice standards in routine pathology laboratories. Thirdly, the targeted therapeutic that is developed needs to specifically and selectively inhibit the target, and as such needs to have a significant anti-cancer effect on the cell (whether it be to inhibit cell proliferation, to induce apoptosis or to enhance sensitivity to other concomitant therapies), in addition to an acceptable toxicity profile. For both endocrine therapies that target the ER, and trastuzumab that targets HER2, each of these three fundamental principles has been adhered to.

Subsequently, it is important that any clinical trials conducted with a targeted therapy demonstrate a significant clinical benefit within an appropriate population of patients. Indeed, if the target is relevant to the disease and the appropriate patients are correctly selected, then the number needed to treat in order to detect a significant effect within a randomised phase II/III clinical trial can be relatively small. This was most clearly seen in the pivotal clinical trial of trastuzumab in HER2-positive metastatic disease in which only 145 patients were needed to show both a highly significant progression-

EGFR = epidermal growth factor receptor; ER = oestrogen receptor; HER2 = human epidermal growth factor receptor $2 ; m$ TOR $=$ mammalian target of rapamycin; $\mathrm{PI} 3 \mathrm{~K}=$ phosphatidylinositol 3-kinase. 
free survival and overall survival benefit for the addition of the monoclonal antibody to paclitaxel chemotherapy as first-line therapy [4]. Likewise, the adjuvant benefit now seen with the appropriate use of tratsuzumab in the targeted population represents a greater than 50\% reduction in the residual risk of recurrence, with an overall survival benefit [5]. Nevertheless, even in selected patients with HER2-positive disease and using a targeted agent, about one-half of patients do not respond - which reflects the complexity of breast cancer.

In contrast, when patients in whom the tumour does not express the relevant target are treated with a molecularly targeted agent, objective response rates are notably disappointing. These disappointing rates were demonstrated recently in the EGF30001 trial of lapatinib, a small-molecule tyrosine kinase inhibitor that targets HER2. It was initially thought that the drug also had significant clinical activity against epidermal growth factor receptor (EGFR), so this trial was established in the first-line metastatic setting for patients with HER2-negative (or unknown) breast cancer [6]. In total 579 patients were randomised to receive 3-weekly paclitaxel with either lapatinib $1,500 \mathrm{mg}$ daily or placebo. A pre-planned retrospective evaluation of HER2 by fluorescence in situ hybridisation identified 86 patients with HER2-positive disease - in this small subgroup, treatment with paclitaxellapatinib resulted in a statistically significant improvement in time to disease progression, objective tumour response and clinical benefit rate. No benefit whatsoever was seen in the HER2-negative group, and no benefit was seen in those patients subsequently analysed for EGFR overexpression [7]. This study has demonstrated very clearly that when the most appropriate patients are selected for a targeted therapy, benefit can be clearly seen in a relatively small number of patients - yet within a wider population who do not express the target within their tumour, these patients have very little to gain from the therapeutic in question.

\section{Issues for clinical trial design of targeted therapies}

For clinical trials with modern targeted therapies it is therefore clear that one size does not fit all, and as such the previous approach involving a classical phase I, phase II, phase III series of trials in unselected patients with metastatic breast cancer is inappropriate.

Once a novel targeted therapy has been developed and has shown promise in various preclinical models, early-phase clinical trials need to be designed to incorporate proof-ofconcept studies that clearly demonstrate the target is being identified within tumour samples, and that a biological effect is occurring following drug exposure in vivo. Early phase I trials need to establish the toxicity profile, the maximum tolerated dose and the recommended dose for phase II studies. Subsequent extension phase $\mathrm{lb} / \mathrm{lla}$ trials need to be conducted in appropriate tumour types with pharmacodynamic endpoints incorporated into the design.
Whether it be tumour or surrogate tissues that are sampled, establishing that the targeted therapeutic is having an effect on the relevant pathways is an important aspect of modern drug development programmes for molecularly targeted therapies. Recently this was clearly demonstrated in breast cancer, with the development of the poly(ADP-ribose) polymerase inhibitor olaparib in a landmark phase I trial, whereby patients with BRCA1 or BRCA2 mutations were enrolled [8]. In this trial, pharmacodynamic studies confirmed poly(ADPribose) polymerase inhibition in tumour tissue and in surrogate samples of peripheral blood mononuclear cells and plucked eyebrow-hair follicles, and the trial showed that antitumour activity was restricted to those mutation carrier patients. These results from a small but very detailed phase I trial have now allowed a rapid development programme to be followed in the most appropriate population of patients.

\section{Molecular profiling of breast cancer}

Once a targeted therapeutic has entered phase II/III development, it remains important to continue evaluation of those patients who derive maximum benefit from the drug. While a novel targeted therapy may have been developed against a growth factor receptor that can be readily be identified by immunohistochemitry or fluorescence in situ hybridisation assays, additional activating mutations either within the target or in associated downstream modulators may account for response or resistance to the targeted therapy in question.

This has been most clearly demonstrated with the anti-EGFR tyrosine kinase inhibitors in nonsmall-cell lung cancer, whereby response to gefitinib is restricted to those with activating EGFR mutations [9] or with increased gene copy number [10]. Likewise in colorectal cancer, the presence of activating downstream KRAS mutations is associated with resistance to the anti-EGFR monoclonal antibody cetuximab, such that testing for this mutation is now recommended so that only those with wild-type KRAS are treated with cetuximab [11]. While overexpression of a given oncogene may identify the best group of patients to treat with a novel targeted therapeutic, only a proportion will probably therefore gain benefit because of other co-existing mutations and molecular alterations that will determine response/resistance to inhibition of the key target (for example, either loss of the PTEN tumour suppressor gene or activating mutation of phosphatidylinositol 3-kinase (PI3K) may modulate response to trastuzumab in HER2-positive breast cancer). Translational studies in samples from treated patients will therefore remain crucial in optimising clinical benefit from these new therapies.

Molecular profiling of tumours is now central to all clinical development programmes for novel targeted therapies, and as such this should further refine and speed up the drug development process as well as provide a more cost-effective approach - both in terms of initial research and development spend but also in ensuring that, once licensed, only those patients who will benefit from the drug are offered it. This is 
well illustrated by the clinical development of mammalian target of rapamycin (mTOR) inhibitors in breast cancer. Following early preclinical promise that these agents may enhance responsiveness to endocrine therapy in ER-positive breast cancer, a large randomised phase III trial of the mTOR inhibitor temsirolimus plus letrozole versus letrozole alone was undertaken in over 1,000 unselected ER-positive postmenopausal women with advanced breast cancer. The trial was halted early because of lack of benefit [12]. The inability to identify patients in whom the tumours may demonstrate dependence on the PI3K/Akt/mTOR pathway being activated may have limited the likelihood of success for this large trial in advanced breast cancer. At a molecular level, concern has also been expressed that mTOR inhibition may release the negative feedback loop via S6kinase and IRS-1 [13], which then enhances further Akt activation and thus overcomes the effects of mTOR inhibition.

An alternative clinical approach to understand which patients may benefit from mTOR inhibition when combined with aromatase inhibitors has been to study biomarker changes in vivo in the preoperative neoadjuvant setting. In a randomised phase II trial in 270 postmenopausal women with ER-positive primary operable breast cancer $(>2 \mathrm{~cm}$ in size), the combination of letrozole $2.5 \mathrm{mg} /$ day and oral everolimus $10 \mathrm{mg} /$ day for 4 months presurgery resulted in a significantly greater tumour shrinkage as judged by ultrasound (58\% vs. $47 \%$, $P=0.03$ ), as well as by clinical and mammographic assessment [14]. Associated correlative science studies were then conducted to determine those tumours most likely to respond to combined mTOR antagonists and an aromatase inhibitor. In particular, elevated levels of one of the downstream biomarkers of mTOR activation (pS6240 kinase) was associated with a greater chance of response to the combination, while specific mutations in PIK3CA were found to be associated with a greater likelihood of an antiproliferative response to the combination of an mTOR antagonist plus letrozole, especially mutations in the small in the allosteric helical domain of exon 9. These types of neoadjuvant studies in primary breast cancer may therefore yield informative and predictive biomarker data that can help select appropriate patients for future clinical trials.

Future studies should therefore make a greater effort to enrich their trial population with the most appropriate patients namely those who have a tumour molecular profile likely to predict benefit from targeting the given pathway. In the absence of the ability to identify the molecular target in samples of metastatic disease (that is, only the original primary tumour is available, as often is the case), understanding the inherent biology in the primary tumour that accounts for relapse/resistance may become crucial is determining which population to select for these studies.

Genomic profiling in ER-positive breast cancer may help identify those more likely to develop resistance to endocrine therapy, or indeed the pathways that these tumours are most likely to utilise as escape mechanisms, which in turn may guide appropriate selection of target therapies to add in at the time of relapse. Clustering tumours together based on oncogenic pathway signatures helps determine both the biology of the disease and the likely response patterns to current conventional therapies [15]. Recent studies have started to show common oncogenic pathways that intrinsic subtypes of breast cancer will utilise, thus allowing strategies to be developed for combinations of various signalling agents to be utilised in an attempt to enhance responsiveness to current therapies [16]. In particular, in ER-positive breast cancer, gene expression profiling has identified in the luminal $B$ subtype that activation of growth factor signalling pathways occurs, often independent of HER2 overexpression, thus contributing to their poorer prognosis [17]. Selection of this subgroup for future combination strategies may yield answers faster than treating a more heterogeneous unselected group of patients with ER-positive breast cancer.

\section{Future directions with novel targeted therapeutics}

There are now a multitude of targeted therapeutics in various stages of clinical development for breast cancer, all based on a rationale that the target in question is valid in the pathogenesis of the disease. While the integration of these therapies with conventional therapeutics has been pioneered by the combination of trastuzumab with chemotherapy, there has been substantial research into combining targeted therapeutics with endocrine therapy to enhance responsiveness and delay resistance [18]. As discussed elsewhere, progress with integrating growth factor receptor tyrosine kinase inhibitors or mTOR antagonists with endocrine therapies has had mixed results in the clinic.

The emphasis is now shifting to targeting networks and pathways with combinations of signalling drugs, either in parallel (so-called combined horizontal blockade) or in series (combined vertical blockade). Selection of which approach is valid depends on key preclinical studies that need to be undertaken in various relevant models, in order to guide which combinations need testing in early-phase clinical trials. Recent preclinical examples of horizontal blockade in breast cancer include mitogen-activated protein kinase kinase (MEK) inhibition combined with PI3K in basal-like breast cancers [19], while vertical blockade includes enhancing responsiveness to HER2directed therapies (trastuzumab, pertuzumab or lapatinib) by combination with a potent PI3K inhibitor [20]. Such preclinical data must then help drive the appropriate early phase I combination studies to be evaluated - studies which if well tolerated and active, as determined by relevant pharmacodynamic endpoints, need to rapidly be tested in phase II studies utilising enrichment strategies to select for the most appropriate breast cancer phenotypes. This will ensure rapid and efficient transition from proof-of-concept studies into pivotal efficacy studies, thus maximising the likelihood of success. 


\section{Conclusion}

Drug development for breast cancer is entering a new phase whereby the number of novel therapeutics available for study will be larger than ever, with an even greater complexity to the clinical and scientific questions being asked. Careful consideration is needed so that several key principles are adhered to, with greater emphasis on preclinical rationale and understanding the biology, real-time molecular profiling and appropriate patient selection, and intelligent clinical trial design that will address the question posed by the novel therapeutic being developed. While the challenges are immense, the opportunities afforded by targeted therapies are enormous - as such, both the pharmaceutical industry and academic investigators have a duty to ensure that we maximise their potential.

\section{Competing interests}

The author has received funding from AstraZeneca and GlaxoSmithKline.

\section{Acknowledgement}

This article has been published as part of Breast Cancer Research Volume 11 Suppl 3 2009: Controversies in Breast Cancer 2009. The full contents of the supplement are available online at http://breastcancer-research.com/supplements/11/S3.

\section{References}

1. Early Breast Cancer Trialists' Collaborative Group: Tamoxifen for early breast cancer: an overview of the randomised trials. Lancet 1998, 351:1451-1467.

2. Romond EH, Perez EA, Bryant J, Suman VJ, Geyer CE Jr, Davidson NE, Tan-Chiu E,Martino S, Paik S, Kaufman PA, Swain SM, Pisansky TM, Fehrenbacher L, Kutteh LA, Vogel VG, Visscher DW, Yothers G, Jenkins RB, Brown AM, Dakhil SR, Mamounas EP,Lingle WL, Klein PM, Ingle JN, Wolmark N: Trastuzumab plus adjuvant chemotherapy for operable HER2-positive breast cancer. N Engl J Med 2005, 353:1673-1684.

3. Piccart-Gebhart MJ, Procter M, Leyland-Jones B, Goldhirsch A, Untch M, Smith I, Gianni L, Baselga J, Bell R, Jackisch C, Cameron D, Dowsett M, Barrios CH, Steger G, Huang CS, Andersson $M$, Inbar $M$, Lichinitser $M$, Láng I, Nitz U, Iwata H,Thomssen C, Lohrisch C, Suter TM, Rüschoff J, Suto T, Greatorex V, Ward C,Straehle C, McFadden E, Dolci MS, Gelber RD; Herceptin Adjuvant (HERA) Trial Study Team: Trastuzumab after adjuvant chemotherapy in HER2-positive breast cancer. N Engl J Med 2005, 353:1659-1672.

4. Slamon DJ, Leyland-Jones B, Shak S, Fuchs H, Paton V, Bajamonde A, Fleming T, Eiermann W, Wolter J, Pegram M, Baselga J, Norton L: Use of chemotherapy plus a monoclonal antibody against HER2 for metastatic breast cancer that overexpresses HER2. N Engl J Med 2001, 344:783-792.

5. Smith I, Procter M, Gelber RD, Guillaume S, Feyereislova A, Dowsett M, Goldhirsch A, Untch M, Mariani G, Baselga J, Kaufmann M, Cameron D, Bell R, Bergh J, Coleman R, Wardley A, Harbeck N, Lopez RI, Mallmann P, Gelmon K, Wilcken N,Wist E, Sánchez Rovira P, Piccart-Gebhart MJ; HERA study te: 2-year follow-up of trastuzumab after adjuvant chemotherapy in HER2-positive breast cancer: a randomised controlled trial. Lancet 2007, 369:29-36.

6. Di Leo A, Gomez HL, Aziz Z, Zvirbule Z, Bines J, Arbushites MC, Guerrera SF, Koehler M, Oliva C, Stein SH, Williams LS, Dering J, Finn RS, Press MF: Phase III, double-blind, ramdomised study comparing lapatinib plus paclitaxel with placebo plus paclitaxel as first-line treatmnet for metastatic breast cancer. $J$ Clin Oncol 2008, 26:5544-5552.

7. Finn RS, Press MF, Dering J, Arbushites M, Koehler M, Oliva C, Williams LS, Di Leo A: Estrogen receptor, progesterone receptor, human epidermal growth factor receptor 2 (HER2), and epidermal growth factor receptor expression and benefit from Lapatinib in a randomised trail of paclitaxel with Lapatinib or placebo as first-line treatment in HER2-negative or unknown metastatic breast cancer. J Clin Oncol 2009, 27:3908-3915.

8. Fong PC, Boss DS, Yap TA, Tutt A, Wu P, Mergui-Roelvink M, Mortimer P, Swaisland H, Lau A, O'Connor MJ, Ashworth A, Carmichael J, Kaye SB, Schellens JH, de Bono JS: Inhibition of poly(ADP-Ribose) polymerase in tumors from BRCA mutation carriers. N Engl J Med 2009, 361:123-134.

9. Paez JG, Jänne PA, Lee JC, Tracy S, Greulich H, Gabriel S, Herman P, Kaye FJ, Lindeman N, Boggon TJ, Naoki K, Sasaki H, Fujii Y, Eck MJ, Sellers WR, Johnson BE, Meyerson M: EGFR mutations in lung cancer: correlation with clinical response to gefitinib therapy. Science 2004, 304:1497-1500.

10. Takano T, Ohe Y, Sakamoto H, Tsuta K, Matsuno Y, Tateishi U, Yamamoto S,Nokihara H, Yamamoto N, Sekine I, Kunitoh H, Shibata T, Sakiyama T, Yoshida T,Tamura T: Epidermal growth factor receptor gene mutations and increased copy numbers predict gefitinib sensitivity in patients with recurrent nonsmall-cell lung cancer. J Clin Oncol 2005, 23:6829-6837.

11. Allegra CJ, Jessup JM, Somerfield MR, Hamilton SR, Hammond EH, Hayes DF,McAllister PK, Morton RF, Schilsky RL: American Society of Clinical Oncology provisional clinical opinion: testing for KRAS gene mutations in patiens with metastatic colorectal carcinoma to predict response to anti-epidermal growth factor receptor monoclonal antibody therapy. J Clin Oncol 2009, 27:2091-2096.

12. Chow LWC, Sun Y, Jassem J, Baselga J, Hayes DF, Wolff AC, Hachemi S, Cincotta M, Yu BW, Kong S, Moore L: Phase 3 study of temsirolimus with letrozole or letrozole alone in postmenopausal women with locally advanced or metastatic breast cancer [abstract 6091]. Breast Cancer Res Treat 2006, 100(Suppl 1):339.

13. O'Reilly KE, Rojo F, She QB, Solit D, Mills GB, Smith D, Lane H, Hofmann F,Hicklin DJ, Ludwig DL, Baselga J, Rosen N: mTOR inhibition induces upstream receptor tyrosine kinase signaling and activates Akt. Cancer Res 2006, 66:1500-1508.

14. Baselga J, Semiglazov V, van Dam P, Manikhas A, Bellet M, Mayordomo J, Campone M, Kubista E, Greil R, Bianchi G, Steinseifer J, Molloy B, Tokaji E, Gardner H, Phillips P, Stumm M, Lane HA, Dixon JM, Jonat W, Rugo HS: Phase II randomised study of neo-adjuvant everolimus plus letrozole compared with placebo plus letrozole in patients with estrogen receptor-positive breast cancer. J Clin Oncol 2009, 27:2630-2637.

15. Bild AH, Yao G, Chang JT, Wang Q, Potti A, Chasse D, Joshi MB, Harpole D,Lancaster JM, Berchuck A, Olson JA Jr, Marks JR, Dressman HK, West M, Nevins JR: Oncogenic pathway signatures in human cancers as a guide to targeted therapies. Nature 2006, 439:353-357.

16. Bild AH, Parker JS, Gustafson AM, Acharya CR, Hoadley KA Anders C, Marcom PK, Carey LA, Potti A, Nevins JR, Perou CM: An integration of complimentary strategies for gene-expression analysis to reveal novel therapeutic opportunities for breast cancer. Breast Cancer Res 2009, 11:R55.

17. Loi S, Sotiriou C, Haibe-Kains B, Lallemand F, Conus NM, Piccart MJ, Speed TP,McArthur GA: Gene expression profiling identifies activated growth factor signaling in poor prognosis (luminal-B) estrogen receptor positive breast cancer. BMC Med Genom 2009, 2:37.

18. Johnston SRD: Enhancing the efficacy of hormonal agents with selected targeted agents. Clin Breast Cancer 2009, 9 (Suppl 1):S28-S36.

19. Hoeflich KP, O'Brien C, Boyd Z, Cavet G, Guerrero S, Jung K, Januario $T$, Savage $H$, Punnoose E, Truong T, Zhou W, Berry L, Murray L, Amler L, Belvin M, Friedman LS, Lackner MR: In vivo antitumour activity of MEK and Phosphatidylinositol 3-kinase inhibitors in basal-like breast cancer models. Clin Cancer Res 2009, 15:4649-4664.

20. Yao E, Zhou W, Lee-Hoeflich ST, Truong T, Haverty PM, EasthamAnderson J,Lewin-Koh N, Gunter B, Belvin M, Murray LJ, Friedman LS, Sliwkowski MX, Hoeflich KP: Suppression of HER2/HER3mediated growth of breast cancer cells with combinations of GDC-0941 PI3K inhibitor, trastuzumab, pertuzumab. Clin Cancer Res 2009, 15:4147-4156. 